\title{
On an Intrinsic Stochastic Fitzhugh: Nagumo Model
}

\section{Elazab NS ${ }^{1,2 *}$}

${ }^{1}$ Department of Mathematics, Cairo University, 9Al Gameya, Oula, Giza Governorate, Egypt

${ }^{2}$ Department of Mathematics, Majmaah University, Al Majmaah, Saudi Arabia

\begin{abstract}
The Fitzhugh-Nagumo model for excitable systems with a high excitation parameter solves the question of selfoscillatory and self-adaptivity in these systems. This is not the case in systems with low excitation parameter. An intrinsic stochastic model that accounts for endogenous fluctuations is proposed. This model solves the question of self-oscillatory and self-adaptivity in systems with low excitation parameter.
\end{abstract}

Keywords: Intrinsic stochasticity; Self adaptivity; Self-Oscillatory; Fitzhugh-Nagumo model

\section{Introduction}

A first model that describes an excitable membrane was proposed by Hodgkin and Huxely $(\mathrm{HH})[1]$. This model solved the question of self-oscillatory in an excitable system that is oscillations between resting and ring membrane potentials, through external inputs from ion channels (or extrinsic noise).

Indeed the system undergoes intrinsic noises from randomness coherent with the processes of opening and closing the ion channels. This had been suggested in recent works [2,3]. A simple model that maintains the main aspects of the $\mathrm{HH}$ model equation had been proposed by Fitzhugh [4] and, Arimoto and Nagumo [5] (FHN). It reads

$$
\frac{d u}{d t}=u(u-a)(1-u)-v, \frac{d u}{d t}=\in(b u-v)
$$

where $u$ is the membrane potential and $\mathrm{v}$ is the recovery current [6$8]$. In the equation $(1), 0<\epsilon<1$, a is the refractory parameter, $0<a<1$, and $b$ is the excitation parameter [9-13]. In case of a high excitation parameter $b, b>(1-a)^{2} / 4$, the eqn. (1) shows an excitable system with a single equilibrium state which is a stable spiral [14-18]. In this case the phase portrait in the uv-plane shows spiraling trajectories. That is in an FHN system with one stable equilibrium state, the question of self-oscillatory was also solved as in the $\mathrm{HH}$ model. Consequently, high excitation is sufficient for self-oscillatory and self-adaptivity [3] in excitable systems.

Numerous studies of the effects of induced-noises in a stable FHN system, coherent to input resonances, had been carried out in the literature [6-18]. In these works induced-noises had been considered either in the activation potential or in the recovery current equations. The phase portrait for stochastic FHN systems shows an induced limit cycle solution. Further, intrinsic stochasticity had been introduced in FHN systems empirically apart from some works [18], where two mechanisms had been suggested. Also, everywhere in the literature it had been assumed that the stochastic noise is Gaussian. We think that, after a recent review in this area [13], the effect of intrinsic stochasticity on a bitable FHN system had not been carried out yet in the literature. This is the case that will be considered here. The mechanism suggested, accounts for endogenous fluctuations in both the activation potential and the recovery current in the absence of external resonances. Which is completely a new mechanism?

We shall present for an approach that an intrinsic stochasticity is induced by the fluctuations in the activation potential and in the recovery current due to the successive opening and closing of channels. Indeed these fluctuations enhance activation near the equilibrium states. This will be clarified later on in theorem 2.1.

In an excitable system with a low excitation parameter $b$, where $b<(1-a)^{2} / 4$. the FHN equation describes a bistable medium where these two stable equilibrium states are $u_{e=e}^{0} v_{e}^{0=0}$ and $u_{e=}^{+}(1+a) / 2+\mathrm{P}(1-a)^{2} / 4-b$, $v_{e}^{+=} b u^{+}$In this case the question of self-oscillatory is not evident by the simple equation (1). It needs further investigations different from those existing in the literature for a FHN system with one stable equilibrium state. The analysis of eqn. (1) shows that when the system starts from near the state of zero potential $u_{e}^{0}$ (resting state), then $\mathrm{u}$ evolutes towards the state $u^{+}$(ring state) stimulated by the recovery current, with $v<0$. While if the initial potential is greater than $a$ then the solution of (1) evolutes towards $u^{+}{ }_{e}$ whatever the behavior of the recovery current. In this case, it was claimed in the literature that the system will return to the state $u_{e}^{0}$ through a long excursion [12]. We think that this do not hold due to the fact that; as the equilibrium states $(0,0)$ and $\left(u^{+}, v_{e}^{+}\right)$are hyperbolic then an FHN system attains these states asymptotically. On the other hand the numerical solution of the eqn. (1) by using RungeKuttamethod does not con rm this statement. In section 3, it will be shown that the solution of eqn. (1) evolutes towards $u^{+}$and does not return to $u_{e}^{0}$. Thus the FHN model with low excitation parameter is not self-adaptive or self-oscillatory.

We think that the system returns to $u_{e}^{0}$ if it is affected by a great stimulus that may arise from endogenous fluctuations (intrinsic noise). Indeed the duration of the potential components in different levels may depend on the strength of the stimulus for intensities near the threshold value. This is accompanied by a long duration of each level (or stage). The duration accounts for the latent period, ring, overshooting, depolarization and hyperpolarization periods. The successive repetition of this sequel may lead to fluctuations in the current. Alternatively, fluctuation in the potential may be argued to the random alteration of the nerve tissue from being a passive conductor to be an active one. Or, fluctuations may be argued to the low threshold of

*Corresponding author: Elazab NS, Department of Mathematics, Majmaah University, Saudi Arabia, Tel: 96616404 4444; E-mail: n.elazab@mu.edu.sa

Received September 19, 2017; Accepted October 13, 2017; Published October 27, 2017

Citation: Elazab NS (2017) On an Intrinsic Stochastic Fitzhugh: Nagumo Model. J Appl Computat Math 7: 376. doi: 10.4172/2168-9679.1000376

Copyright: (c) 2017 Elazab NS. This is an open-access article distributed under the terms of the Creative Commons Attribution License, which permits unrestricted use, distribution, and reproduction in any medium, provided the original author and source are credited. 
excitability of a nerve tissue. We may think that a model that describes the time evolution of an excitable medium may not be deterministic. Due to excitability, a FHN system may undergo fluctuations, so that we may write

$$
u=<u>+\delta u \equiv u+\delta u, v=<v>+\delta v \equiv v+\delta v \text {. }
$$

where $<. . .>$ is an ensemble average over the space of all realizable fluctuations in FHN systems, namely

$$
<\ldots .>\int(\ldots) d m_{s}
$$

and $d m_{S}$ is the measure endowed by this space. We mention that a similar analysis had been carried out for a discrete ensemble of FHN elements $[13,14]$.

In eqn. (2) $\delta u$ and $\delta v$ are the fluctuation about the average with $\langle\delta u\rangle=\langle\delta v\rangle=0$. Hereafter, fluctuations are assumed to be smooth that is $\delta u(t)$ and $\delta v(t)$ are taken to be continuously differentiable functions.

\section{The Model}

By substituting from eqn. (2) into eqn. (1) and by conserving only terms quadratic in $\delta u$, we get [15]

$$
\begin{aligned}
& \frac{d \bar{u}}{d t}+\frac{d \delta u}{d t}=\bar{u}^{3}-3 \bar{u}^{2} \delta u-3 \bar{u}(\delta u)^{2}+(1+a)\left(\bar{u}^{2}+2 \bar{u} \delta u+(\delta u)^{2}\right)-a(\bar{u}+\delta u)-(\bar{v}+\delta v) \\
& \frac{d \bar{v}}{d t}+\frac{d \delta v}{d t}=\in(b \bar{u}-\bar{v})+\in(b \delta u-\delta v)
\end{aligned}
$$

By averaging both sides of (1) over the ensemble, we have

$$
\frac{d<u>}{d t}=-<u^{3}>+(1+a)<u^{2}>-a \bar{u}-\bar{v}, \frac{d \bar{v}}{d t}=\in(b \bar{u}-\bar{v})
$$

By using eqn. (2) into (3) and a direct calculation gives

$$
\begin{aligned}
\frac{d \delta u}{d t} & =p(\bar{u}) \delta u-\delta v, p(x)=-3 x^{2}+2(1+a) x-a \\
\frac{d \delta u}{d t} & =\varepsilon(b \delta u-\delta v)
\end{aligned}
$$

In the eqns. (4) and (5) terms in $(\delta u)^{2}$ and higher were neglected. By the same way the equations for $u$ and $v$ are given by

$$
\begin{aligned}
& \frac{d \bar{u}}{d t}=-\bar{u}^{3}+(1+a) \bar{u}^{2}-a \bar{u}-\bar{v}+(1+a-3 \bar{u})<(\delta u)^{2}> \\
& \frac{d \bar{v}}{d t}=\varepsilon(b \bar{u}-\bar{v})
\end{aligned}
$$

In the eqn. (6), we need to find $\left\langle u^{2}\right\rangle$. To this end we con ne ourselves to the case when the fluctuations in the membrane potential and in the recovery current are decorrelated (decoupled) $\langle\delta v \delta u\rangle=0$. By setting $\sigma_{1}(t)=<(\delta u)^{2>}$ and $\sigma_{2}(t)=<(\delta v)^{2>}$ and by using the eqns. (4) and (5), we find closed form equations for $\sigma_{i}(t)$, namely

$$
\frac{d \sigma_{1}(t)}{d t}=2 p(\bar{u}) \sigma_{1}(t), \frac{d \sigma_{2}(t)}{d t}=-2 \varepsilon \sigma_{2}(t),
$$

and in the eqn. (8) initial conditions are taken $\sigma_{i}(0) \ll b, i=1,2, b$ is the activation parameter, practically $\sigma_{i}(0) \approx b / 10$. We mention that the case when $<\delta v \delta u>6 \neq 0$ will be considered in section 4 . The eqn. (8) integrates to

$$
\sigma_{1}(t)=\sigma_{1}(0) e^{\int^{t} 2 p\left(\bar{u}\left(t_{1}\right)\right) d t_{1}}, \sigma_{1}(t)=\sigma_{1}(0) e^{-2 \varepsilon t}
$$

From the eqn. (9), we and that $\sigma_{2}(t) \rightarrow 0$, when $t \rightarrow \infty$. Also, at the equilibriumstates $u=0$ and $u=u^{+}{ }_{e}$, where $u_{e}^{+}$isgivenin section 1 , weand that $p(0)=-a<0$, and $p\left(u_{e}^{+}\right)=-(1-a)^{2} / 2-b-(1+a) \sqrt{(1-a)^{2} / 4-b<0}$. Thus we have $\sigma_{1}(t) \rightarrow 0$ when $t \rightarrow \infty$ ( as $u \rightarrow 0$ or $\left.u \rightarrow u_{e}^{+}\right)$. Consequently the equilibrium states are unchanged due to fluctuations. Thus in the assumption made in the above, the FHN with intrinsic stochasticity is given by

$$
\begin{aligned}
& \frac{d \bar{u}}{d t}=f(\bar{u}-\bar{v})+q(\bar{u}) \sigma_{1}(t), \frac{d \bar{v}}{d t}=\varepsilon(b \bar{u}-\bar{v}) f \\
& f(x, y)=-x^{3}+(1+a) x^{2}-a x-y, q(x)=1+a-3 x, \sigma_{1}(t)=\sigma_{1}(0) e^{\int j} 2 p\left(\bar{u}\left(t_{1}\right)\right) d t
\end{aligned}
$$

where $p(u)$ is defined in eqn. (6). We analyze the eqn. (7) and prove that, in $0 \leq t<T$, where $T=\max \left\{(b \varepsilon)^{-1}, a^{-1},\left|p\left(u_{e}^{+}\right)\right|^{-1}\right\}$, it describes a selfadaptive system. That is if the system is near the states $u_{e}^{0}=0$ or $u_{e}^{+}$ then fluctuations temporate (increase or decrease) their instantaneous values so that the states $u_{e} \quad$ or $u_{e}$ are no longer stationary.

\section{Theorem 2.1}

The intrinsic FHN eqn. (10) describes a self-adaptive system in 0 $\leq t<T$.

\section{Proof}

We mention that in the absence of the last term in the first eqn. (8), (namely when $\left.\sigma_{1}(0)=0\right)$ and if the system starts from $u=0$ or $\bar{u}=u_{e}^{+}$ the solution in eqn. 10 is $\bar{u}(t)=0$ or $\bar{u}(t)=u_{e}^{+}$respectively as they are the equilibrium states. Now in the presence of fluctuations, we assume the following:

(i) When $\bar{u}(0)=0, \bar{v}=0$ in this case we find that $f(0,0)=0,\left.\frac{d \bar{u}}{d t}\right|_{\bar{u}=0}=(1+a)>0$, and for $t>0$ increases so that. $\frac{d \bar{v}}{d t}>0$ or $\bar{v}=0$.

When $\quad(1+a) / 3<\bar{u}<\left((1+a)+\sqrt{1-a+a^{2}}\right) / 3$ then we find $f(\bar{u}, \bar{v})<0, \mathrm{q}(\bar{u})<0$ and $p(\bar{u})>0$. When $(1+a)+\sqrt{1-a+a^{2}} / 3<\bar{u}<u_{e}^{+}$ we find that $f(\bar{u}, \bar{v})>0, \mathrm{q}(\bar{u})<0$ and $p(\bar{u})<0$ so that $\frac{d \bar{u}}{d t}>0$ for $t>\log (|q(\bar{u})|) / f(\bar{u}, \bar{v}) / 2\left|p\left(\bar{u}_{0}\right)\right|, \bar{u}_{0}=\bar{u}\left(t_{0}\right), 0<t_{0}<t$. Thus $\bar{u}$ attains $u_{e}^{+}$

(ii) When $\bar{u}(0)=u_{e}^{+}, \bar{v}=v_{e}^{+} \quad$ in this case we have $f\left(u_{e}^{+}, v_{e}^{+}\right)=0, q\left(u_{e}^{+}\right)<0$ and $\left.\frac{d \bar{u}}{d t}\right|_{\left(u_{e}^{+}, v_{e}^{+}\right)}<0$, consequently for $\mathrm{t}>0, \frac{d \bar{v}}{d t}<0$ and $\bar{u}$ decreases so that when $\left((1+a)-\sqrt{1-a+a^{2}} / 3<\bar{u}<(1+a) / 3\right.$ then we find that $f(\bar{u}, \bar{v})>0, \mathrm{q}(\bar{u})>0$ and thus $\frac{d \bar{u}}{d t}>0$. When $0<\bar{u}<\left((1+a)-\sqrt{\left.1-a+a^{2}\right)} / 3\right.$ we find that $\mathrm{q}(\bar{u})>0$ and $p(\bar{u})<0$ so that $\frac{d \bar{u}}{d t}<0$ fort $>\log (q(\bar{u}) /|f(\bar{u}, \bar{v})|) / 2\left|p\left(\bar{u}_{0}\right)\right|$ and thus $\bar{u}$ attains the state $u_{e}^{0}=0$. This completes the proofs.

\section{Theorem 2.2}

Theeqn.10describesaself-oscillatorysystemnearthestate $u_{e=}^{0} 0, v_{e}^{0=0}$, in $0<t<T$ where $\operatorname{Tmax}\left\{(b \varepsilon)^{-1}, a^{-1},\left|p\left(u_{e}^{+}\right)\right|^{-1}\right\}$ theorem 2.1 , if the variance of the initial fluctuation satisfies $0<\sigma_{1}(0)-\frac{(a+\varepsilon)(a+b)}{2+a+2 a^{2}-3 b}<2 \sqrt{b \varepsilon}$.

Proof

By linearizing the eqn. (10) near $u_{e}^{0}=0, v_{e}^{0}=0$, the last term in the first eqn. (10); $\sigma_{1}(t)$ becomes $\sigma_{1}(0) e^{-a t}$, and the eqn. 10 becomes

$$
\frac{d \bar{u}^{(1)}}{d t}<-a \bar{u}^{(1)}-\bar{v}^{(1)}+\left(1+a-3 \bar{u}^{(1)}\right) \sigma_{1}(0), \frac{d \bar{v}^{(1)}}{d t}=\varepsilon\left(b \bar{u}^{(1)}-\bar{v}^{(1)}\right)
$$

or

$\frac{d \bar{u}^{(1)}}{d t}<-a \bar{u}^{(1)}-\bar{v}^{(1)}+\left(1+a-3 \bar{u}^{(1)}\right) \sigma_{1}(0), \frac{d \bar{v}^{(1)}}{d t}=\varepsilon\left(b \bar{u}^{(1)}-\bar{v}^{(1)}\right)$ 
According to when $u^{(1)>}(1+a) / 3\left(\right.$ or $\left.u^{(1)>}(1+a) / 3\right)$, respectively. We consider the in eqn. (11) where by using the Grown walls lemma it solves to

$$
\begin{aligned}
& \bar{u}^{(1)}<\frac{(1+a) \sigma_{1}(0)}{a+b}+c_{1} e^{\lambda t}, \bar{v}^{(1)}=\frac{b(1+a) \sigma_{1}(0)}{a+b} c_{2} e^{\lambda t} \\
& \left(a+3 \sigma_{1}(0)+\lambda\right)(\lambda+\varepsilon)+b \varepsilon=0
\end{aligned}
$$

A similar result holds for in eqn. (12).

From the second eqn. (13) a periodic solution exists when

$$
0<\sigma_{1}(0)-\frac{(a+\varepsilon)(a+b)}{2+a+2 a^{2}-3 b}<2 \sqrt{b \varepsilon}
$$

The above equation determines the initial variance of fluctuations in the membrane potential that induce an oscillatory behavior.

After this theorem, we find that an oscillatory solution holds for a sufficiently small initial value of the variance in fluctuations, namely $\sigma_{1}(0)$.

In the next section we shall find numerical solutions of eqn. (10) and show that numerical results do con $\mathrm{rm}$ the above theorems.

\section{Numerical Results}

Our aim here is to solve the eqn. (10) for initial conditions $\bar{u}(0)=u_{0}, \bar{v}(0)=v_{0}$ and hereafter the bar on the variables will be omitted for simplicity. In the first eqn. (10) $v(t)$ is replaced by the formal equation;

$$
v(t)=e^{-\varepsilon t}\left(\varepsilon b \int_{0}^{t} u\left(t_{1}\right) e^{\varepsilon t_{1} d t}+v(0)\right)
$$

We will present for a method for finding approximate analytic solutions of in eqn. (10) [16]. A comparison between this method and some well-known ones is done in some cases. The reason for adopting this method is that it can be applied to find numerical solutions for equations with fluctuations in eqns. $(18,19)$. It is based on using the following steps.

Inspecting the equilibrium points of equations. We have shown that in the case where the fluctuations in the membrane potential and the recovery current is decorrelated, the equilibrium points are not changed due to fluctuations. That is these equilibrium states are; $u_{e=} v_{e=} 0$ and $u_{e=} u_{e}^{+}, v_{e=} b u_{e}$.

By dividing the first eqn. (10) by $\left(u-u_{e}^{0}\right)\left(u-u^{+}{ }_{e}\right)$ and then by integrating formally to get

$$
u(t)=\frac{u_{0} u_{e}^{+}}{u_{0}+\left(u_{e}^{+}-u_{0}\right) e^{\int_{0}^{t} P\left(u\left(\mathrm{t}_{1}\right), v\left(\mathrm{t}_{1}\right), \mathrm{t}_{1} d_{1}\right.}}, P(u, v, t)=\frac{f(u, v)+q(u) \sigma_{1}(t)}{u\left(u-u_{e}^{+}\right)}
$$

where $f(u, v), q(u)$ and $\sigma_{1}(t)$ are given in eqn. (10).

In an analog to the discritization made for finding the fixed point numerically, the eqns. (1) and (2) are written in the form ( for $n>1$ )

$$
u(t)=\frac{u_{0} u_{e}^{+}}{u_{0}+\left(u_{e}^{+}-u_{0}\right) e^{\int_{0}^{t} P\left(u_{n-1}\left(t_{1}\right), v_{n-1}\left(t_{1}\right), t_{1}\right) d d_{1}}}, \mathrm{v}_{n}(t)=e^{-\varepsilon t}\left(\varepsilon b \int_{0}^{t} u_{n}\left(t_{1}\right) e^{\varepsilon t_{1} d t_{t}}+v(0)\right)
$$

For $n=0, u_{0=} u(0)$. For more details [16].

Now we give some numerical solutions of eqn. (1) for initial conditions $u_{0>} a$ and $v_{0>} 0$. Numerical results for the membrane potential calculated by using Runge-Kutta method and by using the method presented in this section for the second approximation, namely $u_{2}(t)$ when $\left.\sigma_{1}(0)=0\right)$ that is in the absence of fluctuations. The results are solid and dotted curves respectively. The specific values of the parameters are given in the legend. The two solutions show the same qualitative behavior for the potential. That is the potential $u(t) \rightarrow u^{+}$, when $t \rightarrow \infty$ and $u(t)$ does not return to the state $u=0$, which does not agree with that claimed [12] (namely the claim that $u(t)$ reaches $u_{e}^{+}$and returns to $u=0$ after a long excursion).

\section{Fluctuations-Coupling Effects}

Here, we consider the effects of coupling between the fluctuations in the membrane potential and the recovery current, namely when $\langle\delta v \delta u\rangle=\sigma_{12}(t) \neq 0$. From the eqn. (4) and (5) the closed form equations for $\sigma_{1}, \sigma_{2}$, and $\sigma_{12}$ are given by

$$
\frac{d}{d t}\left(\begin{array}{l}
\sigma_{1} \\
\sigma_{12} \\
\sigma_{2}
\end{array}\right)=H\left(\begin{array}{l}
\sigma_{1} \\
\sigma_{12} \\
\sigma_{2}
\end{array}\right), H=\left(\begin{array}{ccc}
2 p(\bar{u}) & -2 & 0 \\
b \varepsilon & p(\bar{u})-\varepsilon & -1 \\
0 & 2 b \varepsilon & -2 \varepsilon
\end{array}\right),
$$

where $p(x)=-3 x^{2}+2(1+a) x-a$. It is worth noticing that, in this general case, the FHN intrinsic stochastic model is given the equations in eqn. (21) and equation

$$
\frac{d \bar{u}}{d t}=f(\bar{u}, \bar{v})+q(\bar{u}) \sigma_{1}(t), \frac{d \bar{v}}{d t}=\varepsilon(b \bar{u}-\bar{v})
$$

These five equations have to be solved with initial conditions namely for given $u(0), v(0), \sigma_{1}(0), \sigma_{12}(0)$, and $\sigma_{2}(0)$.

By iteration, the solution of eqn. (21) can be written as

$$
\vec{\sigma}(t)=\left(1+\int_{0}^{t} H\left(t_{1}\right) d t_{1}+\int_{0}^{t} H\left(t_{1}\right) \int_{0}^{t_{1}} H\left(t_{2}\right) d t_{2} d t_{1}+\ldots\right) \vec{\sigma}_{0}
$$

Where $\vec{\sigma}(t)=\left(\sigma_{1}(t), \sigma_{12}(t), \sigma_{2}(t)\right)^{T}$ and $\vec{\sigma}_{0}=\left(\sigma_{1}(0), \sigma_{12}(0), \sigma_{2}(0)\right)^{T}$. We notice that the matrices $H\left(t_{1}\right)$ and $H\left(t_{2}\right)$ do not commute, that is the commutator

$\left[H\left(t_{1}\right), H\left(t_{2}\right)\right]=H\left(t_{1}\right) H\left(t_{2}\right)-H\left(t_{2}\right) H\left(t_{1}\right) \neq 0$. By introducing is the time ordering operator, $\widehat{a} A \hat{A} I J \widehat{O}^{\prime \prime}$ namely

$$
\int_{0}^{t} H\left(t_{1}\right) \int_{0}^{t_{1}} H\left(t_{2}\right) \ldots . \int_{0}^{t_{n-1}} H\left(t_{n}\right) d t_{n} \ldots . . d t_{2} d t_{1}=\widehat{O}\left(\left(\int_{0}^{t} H\left(t_{1}\right) d t_{1}\right)^{n} / \mathrm{n} !,\right.
$$

The eqn. (23) can be written in the form

$$
\vec{\sigma}(t)=\hat{O}\left(\exp \left(\int_{0}^{t} H\left(t_{1}\right) d t_{1}\right) \vec{\sigma}_{0}\right.
$$

Now as

$$
\begin{aligned}
& H(t)=H_{0}+H_{1}(t), H_{0}=\left(\begin{array}{ccc}
-2 a & -2 & 0 \\
b \varepsilon & -\varepsilon-a & -1 \\
0 & 2 b \varepsilon & -2 \varepsilon
\end{array}\right), \\
& H_{1}(t)=\tilde{p}\left(\bar{u}(t)\left(\begin{array}{lll}
2 & 0 & 0 \\
0 & 1 & 0 \\
0 & 0 & 0
\end{array}\right)\right.
\end{aligned}
$$

where $\tilde{p}(x)=-3 x^{2}+2(1+a) x$.

By using Zassenhaus formula [17] for non -commutative matrices

$$
\begin{aligned}
& \vec{\sigma}(t)=\exp \left(\mathrm{H}_{0} \mathrm{t}\right) \exp \left(\theta \mathrm{H}_{1}\right) \exp \left(-C_{2} / 2 !\right) \exp \left(C_{3} / 3 !\right) \ldots \vec{\sigma}_{0} \\
& \left.C_{2}=\left[\mathrm{tH}_{0}, \theta \mathrm{H}_{1}\right]=t \theta\left[\mathrm{H}_{0} \mathrm{H}_{1}-\mathrm{H}_{1} \mathrm{H}_{0}\right], \mathrm{C}_{3}=2\left[\theta \mathrm{H}_{0}, \theta \mathrm{H}_{1}\right]\right]+ \\
& {\left[\mathrm{tH}_{0},\left[\mathrm{tH}_{0}, \theta \mathrm{H}_{1}\right]\right], \theta=\int_{0}^{t} p\left(\bar{u}\left(t_{1}\right)\right) d t_{1}}
\end{aligned}
$$

By considering the norm of the commutators $\left\|C_{i}\right\|_{2}=\max _{j} \sqrt{\left|\lambda_{i j}\right|}$ where $\lambda_{i j}$ are the eigenvalues of the matrix $C_{i} C_{i}^{T}$. When $i=2,3$ we find that $\left\|C_{2}\right\|_{2}<4 t|\theta(t)| b \varepsilon$ and $\left\|C_{3}\right\|_{2}<2^{3} t^{2}|\theta(t)|(b \varepsilon)^{2}$. To carry out numerical computations we use the eqn. (27) by neglecting $C_{3}$ and higher limiting calculations for $t<\left(9 / 4(b \varepsilon(1+a))^{2}\right)^{-1 / 3}$ as $|\theta(t)|<t(1+a)^{2} / 3$. Numerical 
results for the membrane potential, recovery current, mean square of the fluctuations in the potential and recovery current and the mean of the correlated fluctuations in both are displayed in the same initial conditions as respectively.

\section{Conclusions}

We have constructed an intrinsic stochastic FHN-model, for systems with low excitation parameter that accounts for endogenous fluctuations. A closed form for the set of equations for the ensemble averages of the membrane potential, recovery current and variances in their fluctuations had been given in eqns. (22) and (21). Theoretical proofs had shown that a system, which is described by this model, is self-adaptive and self-oscillatory. Numerical results had been carried out by including fluctuations effects and they confirmed the theoretical predictions. Consequently this model conserves the main features as in an excitable system with high excitation parameter. The model presented, accounts for fluctuations about the mean and it may be considered as a simple model for describing smooth-noisy systems.

\section{References}

1. Hodgkin AL, Huxley AF (1952) A quantitative description of membrane current and its application to conduction and excitation in nerve. The Journal of Physiology 117: 500-544

2. Deflice LJ (2000) Linear analysis in non-linear systems; signal to noise ratios in noisy neurons. Chaos Solitons \& Fractals 11: 1823-1826.

3. Vajtai R, Kiss LB, Gingle Z (2000) A self-adaptive stochastic resonator with logarithmic transfer. Chaos Solitons \& Fractals 11: 1933-1935.

4. Fitzhugh R (1961) Impulses and physiological states in theoretical models of nerve membrane. Biophysical Journal 1: 445-466.
5. Arimoto S, Nagumo JS, Yoshzawa S (1962) An active pulse transmission line simulating nerve axon. Proceedings of the IEEE 50: 2061-2071.

6. Lindner B, Schimansky-Geier L (2000) Physical Review E 61: 6103.

7. Collins JJ, Chow CC, Imho TT (1995) A periodic stochastic resonance. Physical Review E52, R 3321-3324.

8. Pei XA, Bachman K, Moss F (1995) The detection threshold, noise and stochastic resonance in the Fitzhugh-Nagumo neuron model. Physics Letters A 206: 61-65.

9. Longtin A (1993) Stochastic resonance in neuron models. Journal Statistical Physics 70: 309-327.

10. Chilov DR, Longtin A, Muller-Gerking J (1997) Stochastic resonances of neural ensembles. Physical Review E 55: 1798-1808.

11. Longtin A, Chilov DR (1998) Stochastic and deterministic resonances in excitable systems. Physical Review Letter 82: 4012.

12. Murray JD (1993) Mathematical Biology. (2 ${ }^{\text {nd }}$ edn.), Springer.

13. Lindner B, Garcia-Ojalvo J, Neiman AB, Schimansky-Geier L (2004) Effects of noise in excitable systems. Physical Review 392: 321-424.

14. Zaks MA, Neiman AB, Feisted S, Schimansky-Geier L (2003) Physical Review E 68: 196-206.

15. Birmingham TJ, Bornatici M (1972) Resonant diffusion in the presence of strong plasma turbulence. Phys. Fluids. 4: 1778-1789.

16. Abdel-Gawad $\mathrm{HI}$ (2008), On a finite memory transport in the Fisher equation. Applied Mathematical Model 32: 1883-1893.

17. Brian CH (2003) Lie group Lie algebra and representation. An elementary introduction Springer.

18. Tang J, Jia Y, Yi M, Ma J, Li J (2008) Multiplicative-noise-induced coherence resonance via two mechanisms in bistable neural models. Physical Review E 77: 601905 . 